\title{
Synthesis and Characterization of Nanocomposite Hydroxy- Sodalite/Ceramic Membrane via Pore-Plugging Hydrothermal Synthesis Technique
}

\author{
M.O. Daramola*, A. Dinat and S. Hasrod
}

\begin{abstract}
School of Chemical and Metallurgical Engineering, Faculty of Engineering and the Built Environment, University of the Witwatersrand, Private Bag X3, Wits 2050, Johannesburg, South Africa
\end{abstract}

\begin{abstract}
In this article, synthesis and characterization of nanocomposite hydroxy sodalite (H-SOD)/a-alumina membrane via the so-called "pore-plugging" hydrothermal synthesis (PPH) protocol is reported for the first time. In nanocomposite architecture membranes, zeolite crystals are embedded within the pores of the supports instead of forming thin-film layers of the zeolite crystals on the surface of the supports. The as-prepared membranes were characterized with SEM and FTIR for morphology and purity of the H-SOD crystals. Compared to the conventional in-situ direct hydrothermal synthesis, membranes obtained from $\mathrm{PPH}$ possess higher mechanical and thermal stability. In addition, defect control with nanocomposite architecture membrane is possible because the zeolite crystals are embedded within the support pores of the membrane, thereby limiting the maximum defect size to the pore sizes of support. The nanocomposite architecture nature of the membranes safeguards the membrane from shocks or abrasion that could promote defects/inter-crystalline pores formation. These advantages could be helpful in the scale-up process of the preparation procedure of membranes at the commercial level with less-demanding conditions.
\end{abstract}

Keywords: Hydroxy sodalite, Nanocomposites, Membranes, Ceramics, Porous materials, Thin films.

\section{INTRODUCTION}

Most often, zeolite membranes are prepared by conventional one-step direct or in situ hydrothermal synthesis in an autoclave under autogeneous pressure. In this one-step process, nucleation and growth processes take place in the presence of a support. However this simple strategy presents some drawbacks such as competition between nucleation and crystal growth process, limiting the nuclei density due to mass-transfer limitations. Therefore obtaining continuous and well inter-grown zeolite layer on the supports using this method necessitates growing thick zeolite films on the supports. Furthermore, the number and distribution homogeneity of the zeolite particles on the support depend on a number of parameters, such as the surface properties, that are difficult to control.

Using the conventional in situ direct hydrothermal synthesis technique, hydroxy sodalite (H-SOD) membranes prepared on asymmetric a-alumina- $\mathrm{TiO}_{2}$ disks (average pore size distribution of the $\mathrm{TiO}_{2}$ top layer $80 \mathrm{~nm}$ ) have been reported [1-3]. The synthesized membranes consisted of thin-films of $\mathrm{H}$ SOD particles on the supports. The performance of the as-prepared membranes was evaluated in series of industrial processes like dewatering of alcohols, dehydrating of organic acids and desalination of

*Address correspondence to this author at the School of Chemical and Metallurgical Engineering, Faculty of Engineering and the Built Environment, University of the Witwatersrand, Johannesburg, South Africa;

Tel: +27117177536; E-mail: Michael.daramola@wits.ac.za seawater [1-3]. Hydroxy sodalite (H-SOD) is a crystalline and hydrophilic zeolite type made of cubic array of $\beta$-cages as primary building block $[4,5]$. H-SOD has cage dimension of $\sim 0.265 \mathrm{~nm}$, making it a good candidate for separating mixtures that contain small molecules such as water $(\sim 0.27 \mathrm{~nm})$, helium $((\sim 0.26$ $\mathrm{nm})$ and ammonia $((\sim 0.25 \mathrm{~nm})$ [5]. Furthermore, potential applications of these membranes were demonstrated in the esterification-coupling reaction and separation process for selective water removal from product mixture [6,7]. In addition, these membranes could find potential applications in Fischer-Tropsch (FT) process and high-pressure boilers for water removal [7].

For industrial applications of these membranes, high flux defect-free membranes with ultra-thin membrane layers are required. Furthermore, membrane configurations such as tubular and hollow fibres are preferred to the disk-type. In comparison with conventional single tube geometries, hollow fibres have larger surface area-to-volume ratios $\left(>3000 \mathrm{~m}^{2} / \mathrm{m}^{3}\right)$ [8]. In addition, several hollow fibres can be made into fibre bundles, reducing both the size and cost of permeating modules for industrial processes. However to form defect-free H-SOD membranes on hollow fibre or tubular supports having larger pore size distribution greater than $80 \mathrm{~nm}$ using conventional direct hydrothermal synthesis, several cycles of hydrothermal synthesis will be required. Multi-stage conventional direct hydrothermal synthesis results in formation of thicker membrane layers, thereby reducing dramatically 
the membrane fluxes. In addition, thermal expansion mismatch between the membrane layer and the support could occur, thereby promoting formation of cracks which results in loss of membrane selectivity.

Nanocomposite architecture membranes synthesized through "pore-plugging" hydrothermal technique (PPH) might alleviate the aforementioned challenges. Advantages of nanocomposite architecture membrane compared to "film-like" counterpart include defect control and higher mechanical and thermal stability. Defect control with nanocomposite architecture membrane is possible because the zeolite crystals are embedded within the support pores of the membrane, thereby limiting the maximum defect size to the pore sizes of support [9]. Furthermore, the nanocomposite architecture safeguards the membrane from shocks or abrasion that could promote defects/inter-crystalline pores formation [9]. The unique characteristics of the nanocomposite architecture could facilitate membrane handling and module assembling, in particular, when a catalyst is placed in contact with the membrane for catalytic membrane applications [10]. Also, when large membrane areas are required, pore-plugging synthesis route appears more dependable because an initial imperfection will not originate a larger defect that will be larger than the pore size of the substrate. This advantage could be helpful in the scale-up process of the preparation procedure of $\mathrm{H}$-SOD membranes at the commercial level with lessdemanding conditions [11].

Outstanding performance of nanocomposite MFI zeolite membranes synthesized on $\alpha$-alumina supports via the "pore-plugging" hydrothermal technique has been reported for xylene isomer separation and $\mathrm{CO}_{2} / \mathrm{N}_{2}$ separation when compared to their "film-like" counterpart [12,13]. Considering the aforementioned merits of nanocomposite architecture membranes over their "film-like" counterparts, this study investigated the synthesis and characterization of nanocomposite $\mathrm{H}$ SOD/ a-alumina membranes. As far as could be ascertained, this is the first report of such in the open literature.

\section{EXPERIMENTAL}

\subsection{Membrane Support}

The membranes used for this study was prepared via the "pore-plugging" synthesis technique on asymmetrical Pall-Exekia $\alpha$-alumina tubes (o.d. $10 \mathrm{~mm}$, i.d. $7 \mathrm{~mm}$, length $5 \mathrm{~cm}$ ). The cross-sectional layers of the support have dimensions: outer layer, $1200 \mathrm{~nm}$; intermediate layer, $800 \mathrm{~nm}$; inner layer, $200 \mathrm{~nm}$. Prior to membrane synthesis, the supports were cleaned in boiling water for 1-2 hours to remove dirt and loose particles from their pores.

\subsection{Membrane Synthesis and Characterization}

Anhydrous sodium metasilicate $\left(\mathrm{Na}_{2} \mathrm{SiO}_{3}\right)$, sodium hydroxide $(\mathrm{NaOH})$, anhydrous sodium aluminate $\left(\mathrm{NaAlO}_{2}\right)$ and de-ionized water were used to prepare the synthesis solution. These materials were mixed together in a polytetrafluoroethylene (PTFE) bottle and stirred for 1 hour on a magnet stirrer to yield a precursor mixture of molar composition ratio: $5 \mathrm{SiO}_{2}: \mathrm{Al}_{2} \mathrm{O}_{3}: 50 \mathrm{Na}_{2} \mathrm{O}: 1005 \mathrm{H}_{2} \mathrm{O}$. For the $\mathrm{PPH}$ hydrothermal synthesis, $\sim 45 \mathrm{~mL}$ of the vigorously mixed precursor solution was poured into a Teflon-lined stainless steel autoclave and the cleaned support was placed in it (see Figure $\mathbf{1}$ for schematic illustration). For the formation of nanocomposite membranes via $\mathrm{PPH}$, the temperature programme depicted in Figure $\mathbf{2}$ was used. At the end of the hydrothermal synthesis, the asprepared membrane was washed thoroughly with deionized water until the $\mathrm{pH}=7-8$. Also the $\mathrm{H}-\mathrm{SOD}$ crystals at the bottom of the Teflon-lined autoclave was collected and washed. The washed membrane and the crystals were dried overnight at $373 \mathrm{~K}$ in an oven under the flow of nitrogen. The morphology of the cleaned support, the as-prepared nanocomposite membrane and that of the H-SOD crystals were inspected by SEM (JSM-5800LV, $20 \mathrm{kV}$ ) coupled with EDX analysis (Edax Phoenix, 1- $\mu \mathrm{m}$ microprobe), and Fourier Transform Infrared (FTIR) analysis of the crystals was conducted with Bruker IFS spectrometer using $\mathrm{KBr}$ pellets as background to obtain infrared spectrum that could provide further information about the purity of the $\mathrm{H}$ SOD crystals. Crystal phases in the as-prepared samples were identified by X-ray diffraction (XRD) (model: Bruker D8 advance X-ray diffractometer) using Co Ka radiation $(\lambda=0.179 \mathrm{~nm})$ at a scan rate of 0.25 sec/step and a step size of $0.02^{\circ}$.

\section{RESULTS AND DISCUSSION}

\subsection{Membrane Synthesis and Characterization}

The SEM micrographs showing the cross-sectional view of the support are depicted in Figure 3. The SEM micrographs and the FTIR spectrum of the H-SOD particles obtained from the bottom of the Teflon-lined autoclave are presented in Figure 4. The EDX analysis of the H-SOD crystals showed that $\mathrm{Si} / \mathrm{Al}=1-1.5$, 


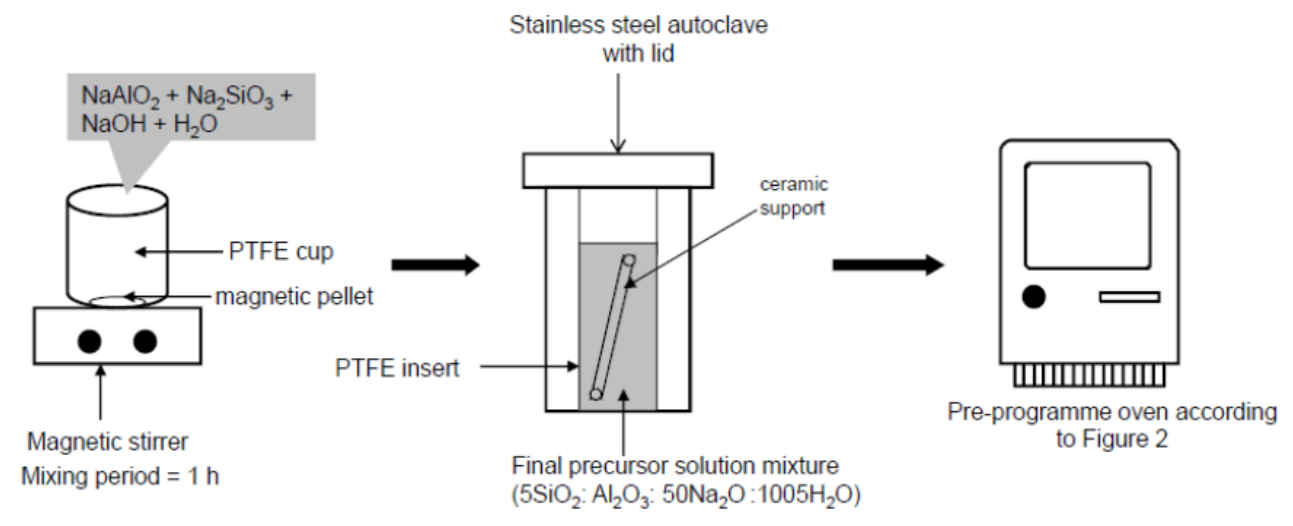

Figure 1: Schematic of the procedure for "pore-plugging" membrane synthesis.

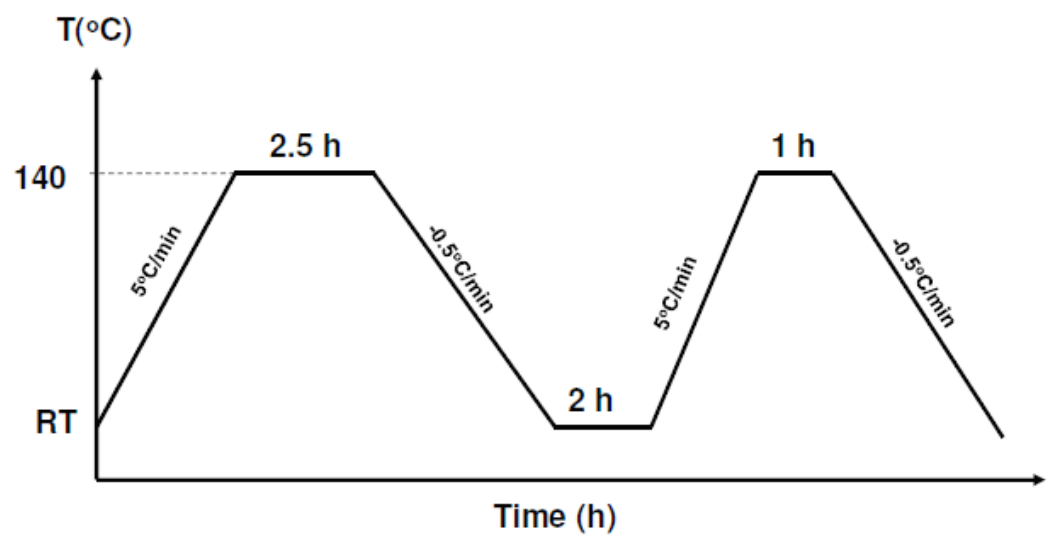

Figure 2: Temperature programme for the "pore-plugging" hydrothermal synthesis. RT: room temperature.

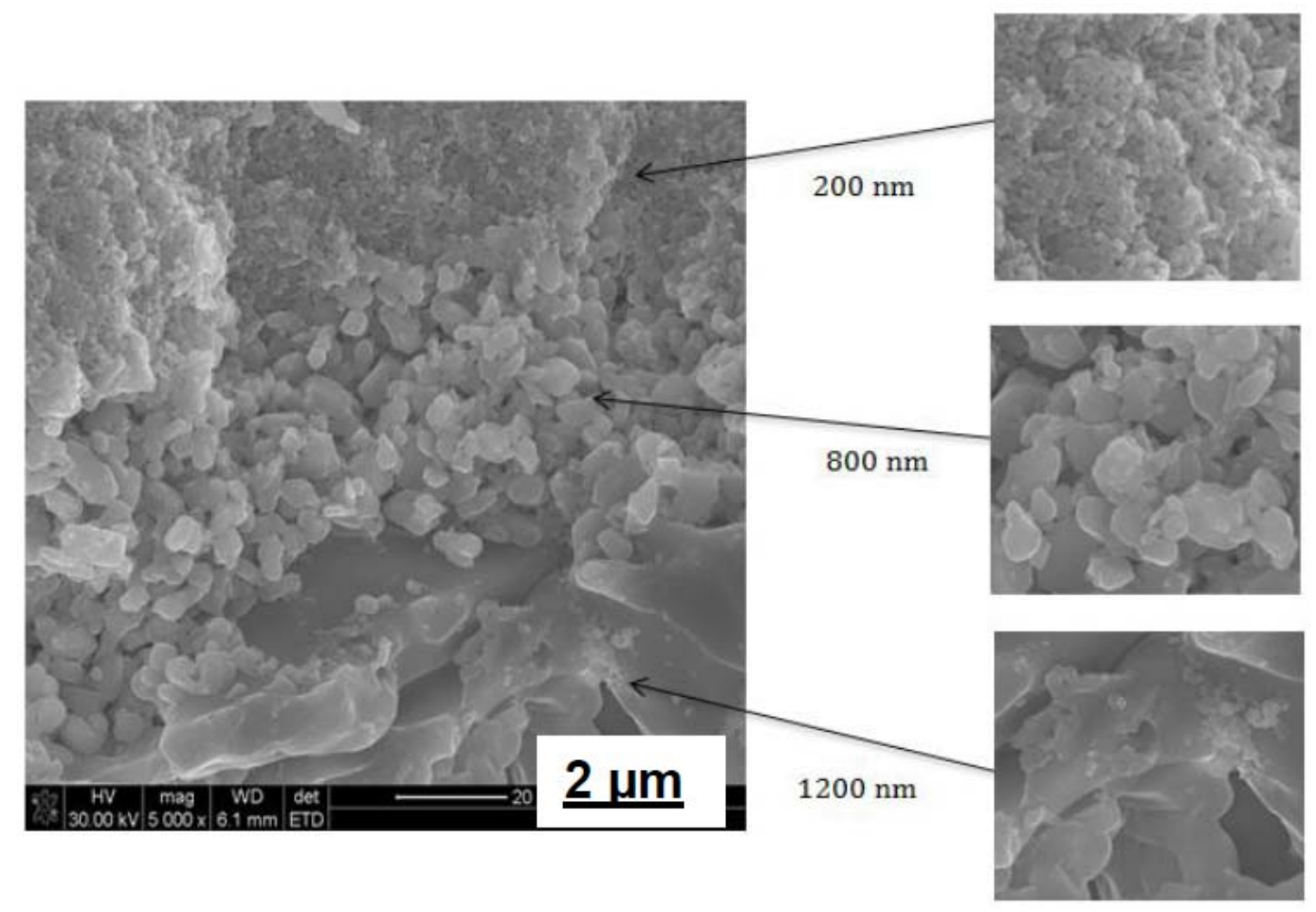

Figure 3: Physico-chemical characterization of the support. SEM micrographs of the support showing the three layers (200 nm, $800 \mathrm{~nm}$ and $1200 \mathrm{~nm}$ ). 

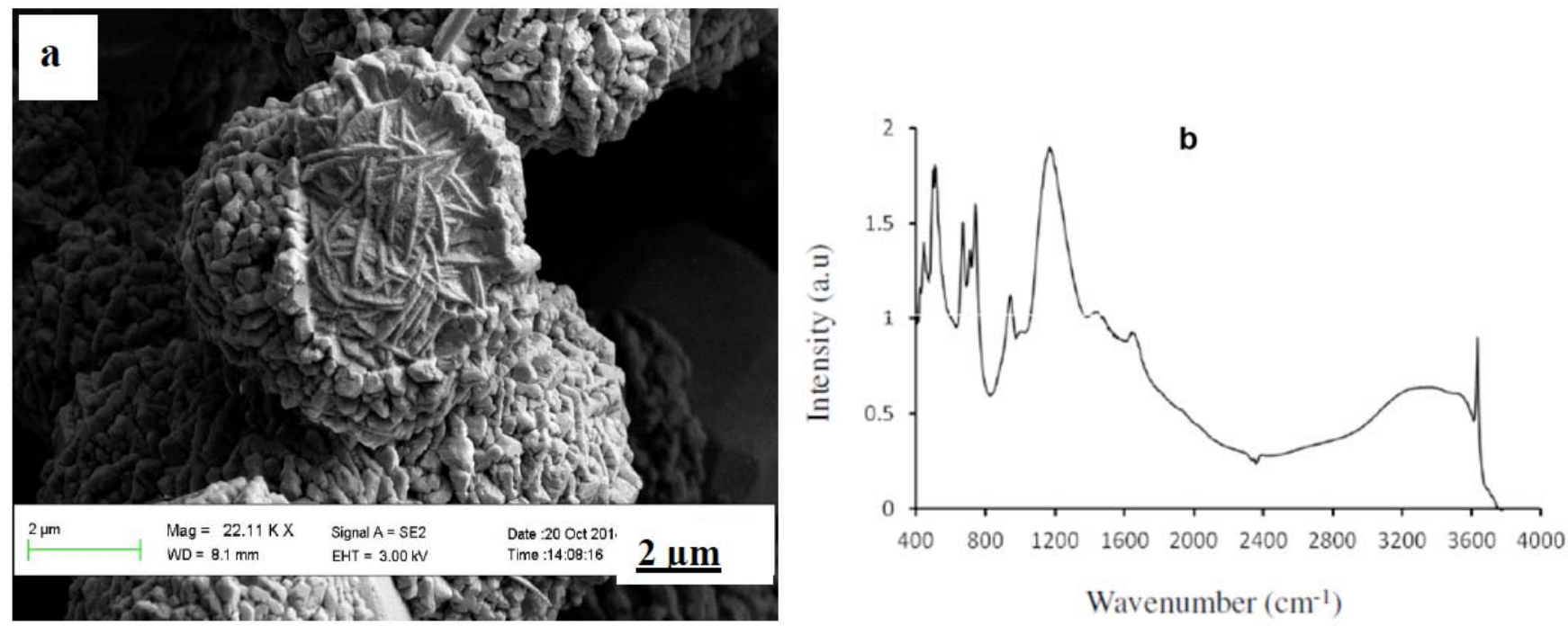

Figure 4: (a) SEM micrograph showing different morphologies of H-SOD crystals; (b) FTIR spectrum confirming formation of HSOD crystals from PPH.

indicating $\mathrm{H}-\mathrm{SOD}$ framework. However, a mixture of $\mathrm{H}$ SOD particles with cubic shape and nanorod-like shape was formed (see Figure 4). The observed morphology is consistent with previous studies where other shapes of hydroxy sodalite such as nanorods, flower-like, urchin, thread-ball and cubic/octahedral shape have been reported [14-16]. These different shapes were observed also in the synthesized nanocomposite membranes (see Figure 5). Results obtained in this study confirm also interplay between the growth process and the nucleation process that seems to proceed in parallel once high temperature is applied [17]. The FTIR spectrum in Figure 4 shows the strong broad band centered at $\sim 1000 \mathrm{~cm}^{-1}$ that can be attributed to the asymmetric stretching vibration of T-O$\mathrm{T}(\mathrm{T}=\mathrm{Si}, \mathrm{Al})$ and those appeared around 740 and 660 $\mathrm{cm}^{-1}$ can be assigned to the symmetric stretching vibration of T-O-T. In addition, the strong broad band centered at $\sim 3600 \mathrm{~cm}^{-1}$ can be assigned to the presence of water molecules in the framework of the synthesized crystals [5]. Regarding the formation of pure hydroxy sodalite particles, the information obtained from the infrared spectrum of the H-SOD crystals is consistent with literature $[15,18]$.

Figure 5 shows the SEM micrographs of different views of the as-prepared nanocomposite $\mathrm{H}$ SOD/alumina membrane. The SEM micrograph in Figure 5 shows vividly the distinct cross-sectional layers of the support. Figure 5a shows the formation of $\mathrm{H}$-SOD particles crystals within the 3 layers of the support. Figure 5a shows that all the three layers contain sodalite particles and the $200 \mathrm{~nm}$-layer of the support seems totally plugged with the zeolite crystals. In addition, Figure $\mathbf{5 b}$ shows that the $800 \mathrm{~nm}$-layer of the support contain a lot of the sodalite crystals but not totally plugged when compared to the $200 \mathrm{~nm}$-layer and the $1200 \mathrm{~nm}$-layer has a few crystals embedded within it (see Figure 5c). The total pore-plugging of the $200 \mathrm{~nm}$-layer indicates that the membrane might be defect free. Although measurement of transport performance, such as quick permeation test and mixture gas separation, is required to confirm this speculation. In addition, the EDX analysis shows an average Si/A1 ratio of 1-1.5 (semi-quantitative analysis) on the inner active layer, corresponding to the expected Si/Al ratio for $\mathrm{H}$-SOD framework $[4,5]$.

\subsection{Mechanism of the Formation of Nanocomposite Architecture}

In-depth understanding of the mechanism involved in the formation of the crystals and the nanocomposite architecture could pave a way for the optimization and scaling-up of the synthesis protocol. Hence, investigation of the mechanism of crystal formation was carried out. During the investigation, two autoclaves were put in the pre-heated oven during the synthesis (see Figure 6). Then, the autoclaves were removed from each of the stages and the crystals obtained thereof were washed until pH of 7 and dried at $120^{\circ} \mathrm{C}$ in an oven. Morphology, crystallinity and purity of the crystals were checked using SEM and XRD according to the conditions discussed in section 2.2.

The XRD patterns and the SEM images depicted in Figures $\mathbf{7}$ and $\mathbf{8}$, respectively, indicate formation of 

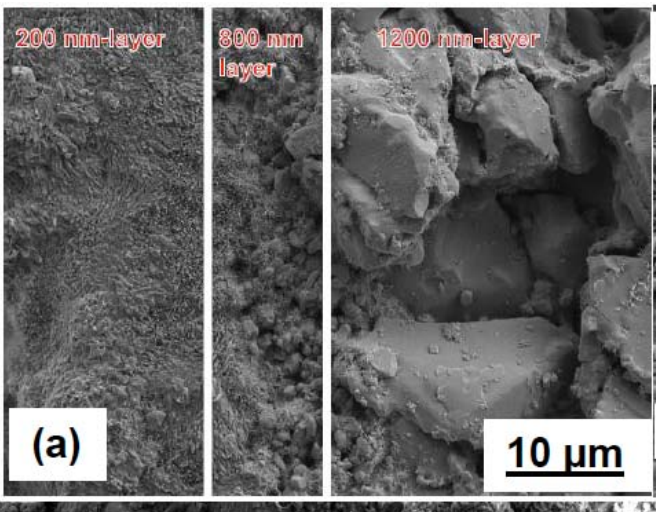

(c)

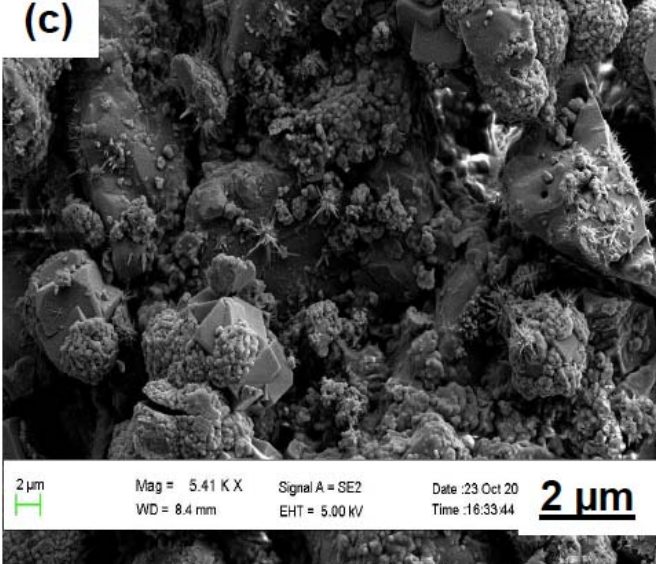

(b)

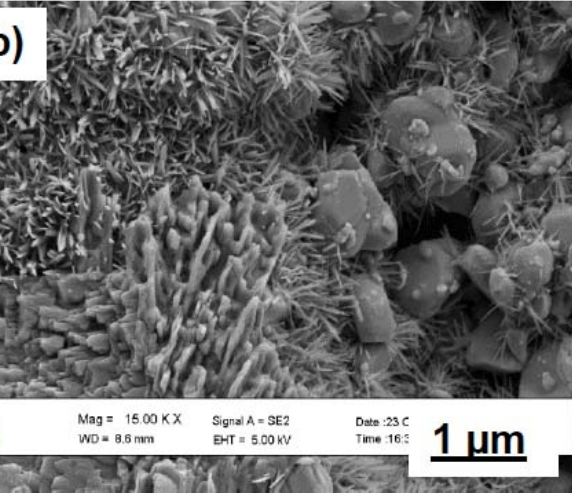

(d)

Figure 5: SEM micrographs of the nanocomposite H-SOD/ alpha-alumina membrane. Cross sectional view of the membrane showing pore-plugged 3 layers (a); pore-plugged $200 \mathrm{~nm}$ and less pore-plugged $800 \mathrm{~nm}$-layer (b); H-SOD particles in the 1200 nm-layer (c); surface view of the 200 nm-layer (d).

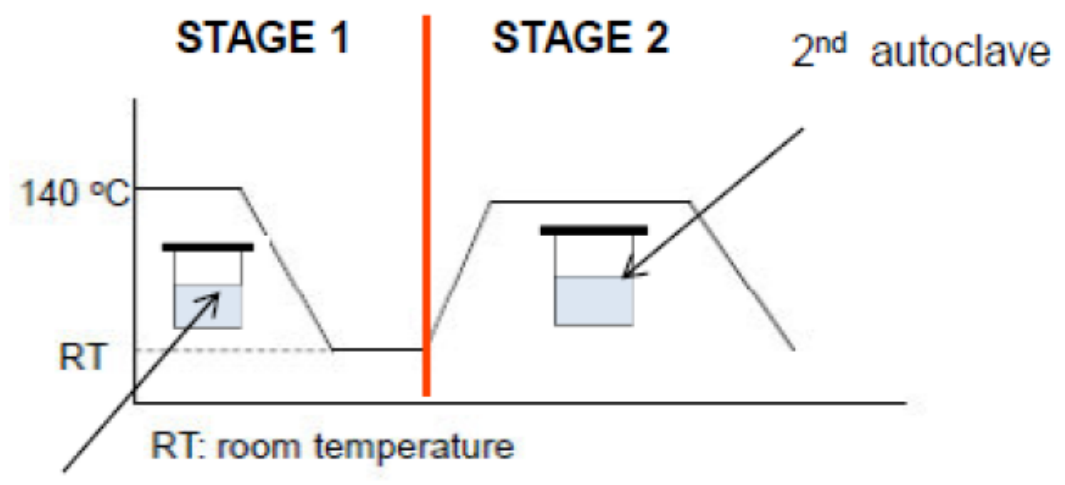

\section{$1^{\text {st }}$ autoclave}

Figure 6: Temperature programme adopted during the investigation of mechanism involved in the nanocomposite formation.

pure H-SOD crystals at both stages (pre-interruption and post-interruption stages). Furthermore, the average crystal size at pre-interruption and postinterruption stages, as inferred from SEM images, were $\leq 2 \mu \mathrm{m}$ and $\sim 5 \mu \mathrm{m}$, respectively. Based on these findings, it could be speculated that primary nucleation occurs at pre-interruption stage, resulting into formation of smaller crystallites. The presence of smaller crystallites (obtained from pre-interruption stage) stimulates secondary nucleation, resulting in enhanced crystallization rates during post-interruption stage. The increase in the crystallization rates results into increasing growth rate, hence formation of larger crystallites during the post-interruption stage. The enhanced crystallization was attributed to: (i) the enhanced rate at which solute integrates into the solid 


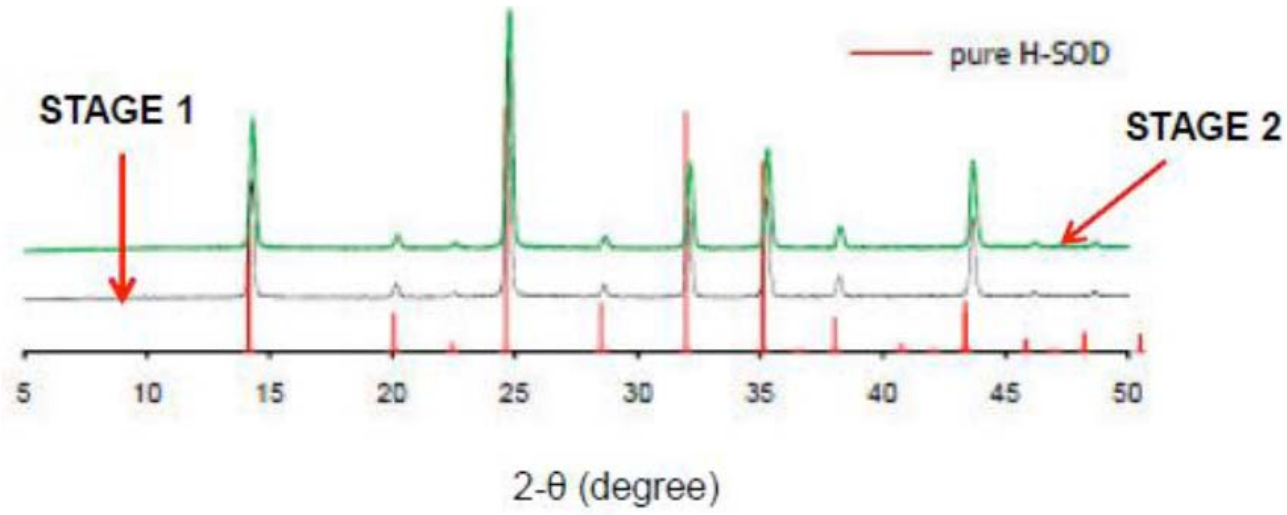

Figure 7: XRD patterns of the synthesized H-SOD crystals via $\mathrm{PPH}$ at different temperature programs: (stage 1: pre-interruption stage, stage 2: post-interruption stage).

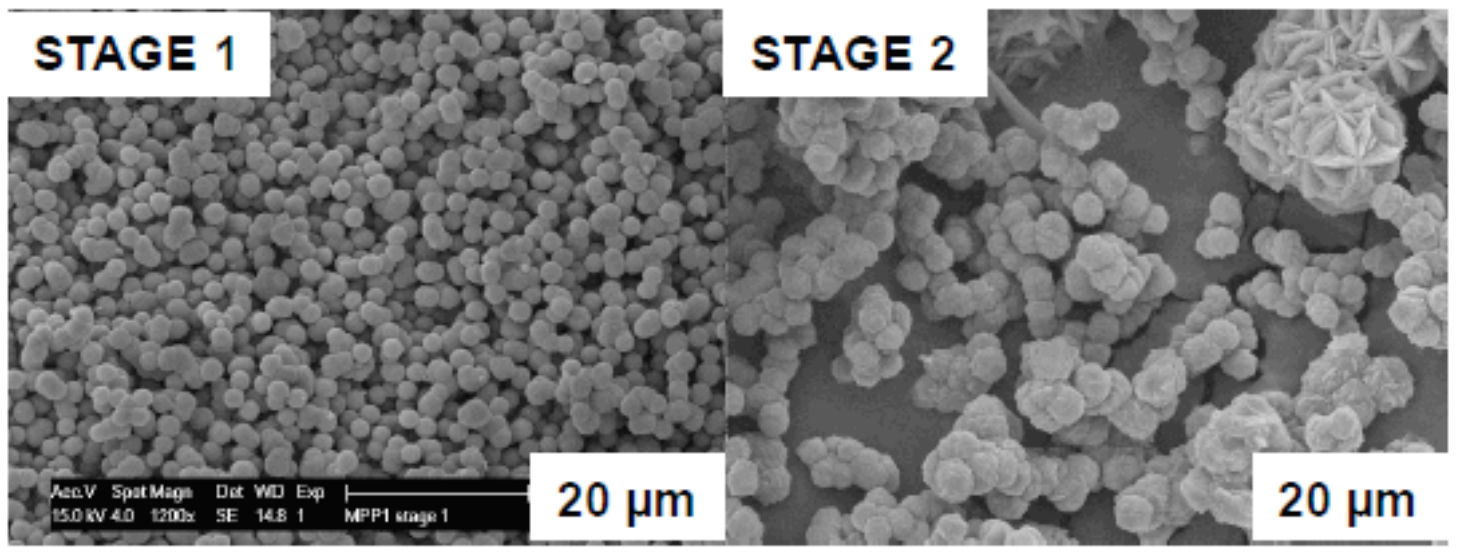

Figure 8: SEM images of the synthesized H-SOD crystals. Stage 1: pre-interruption stage; stage 2: post-interruption stage).

phase from the precursor solution as a result of the increased surface area due to the presence of the seeds and, (ii) enhanced nucleation of new crystals [19]. Therefore, formation of the nanocomposite architecture could be attributed to the heterogeneous nucleation during the temperature interruption. The presence of smaller crystallites (obtained from preinterruption stage) stimulates secondary nucleation, resulting in enhanced crystallization rates during postinterruption stage $(1 \mathrm{~h})$. The increase in the crystallization rates results into increasing growth rate, hence formation of larger crystallites, which eventually pore-lugged the support pores, during the postinterruption stage. This is in agreement with the explanation provided by $\mathrm{Li}$ et al. [9] for the formation of nanocomposite MFI membranes supported on ceramics.

\section{CONCLUSION}

In this study, pore-plugging hydrothermal synthesis technique was used to synthesis nanocomposite $\mathrm{H}$ SOD/a-alumina membranes. The SEM micrographs of the as-prepared membranes reveal that nanocomposite $\mathrm{H}$-SOD/a-alumina membranes were successfully synthesized. As far as we could ascertain, this is the first report on the synthesis of nanocomposite hydroxy sodalite/a-alumina using "pore-plugging" hydrothermal synthesis technique. The encouraging results documented in this article could serve as an impetus for the application of the poreplugging hydrothermal synthesis technique for the production of defect-free supported H-SOD membranes, which can be applied for selective water removal in process industries.

\section{ACKNOWLEDGEMENT}

MOD acknowledges Prof. Kapteijn and A/Prof. Gascon of the Delft University of Technology, The Netherlands, for the opportunity to gain from their wealth of experience in the membrane field.

\section{REFERENCES}

[1] Khajavi S, Jansen JC, Kapteijn F. In: From zeolite to Porous MOF Materials, Xu R, Gao Z, Chen J, Yan W, Eds. Elsevier, The Netherlands 2007; pp. 1028-1035. 
[2] Khajavi S, Jansen JC, Kapteijn F. Production of ultra pure water by desalination of sea water using a hydroxy sodalite membrane. J Membr Sci 2010; 356: 52-57. http://dx.doi.org/10.1016/j.memsci.2010.03.026

[3] Khajavi S, Jansen JC, Kapteijn F. Application of hydroxy sodalite films as novel water selective. J Membr Sci 2009; 326: 153-160.

http://dx.doi.org/10.1016/j.memsci.2008.09.046

[4] Khajavi S, Kapteijn F, Janssen JC. Synthesis of thin defectfree hydroxy sodalite membranes: New candidate for activated water permeation. J Membr Sci 2007; 299: 63-72. http://dx.doi.org/10.1016/j.memsci.2007.04.027

[5] Breck DW. Zeolite Molecular Sieves: Structure, Chemistry and Use, USA, New York: Wiley 1974

[6] Khajavi S, Jansen JC, Kapteijn F. Application of a sodalite membrane reactor in esterification-coupling reaction and separation. Catalysis Today 2010; 156: 132-139. http://dx.doi.org/10.1016/i.cattod.2010.02.042

[7] Daramola MO, Aransiola EF, Ojumu TV. Potential Applications of Zeolite Membranes in Reaction Coupling Separation Processes. Materials 2012; 5: 2101-2136. http://dx.doi.org/10.3390/ma5112101

[8] Daramola MO, Burger AJ, Pera-Titus $M$, Giroir-Fendler A, Lorenzen L, Miachon S, Dalmon J-A. Nanocomposite MFI hollow-fibre membranes via pore-plugging synthesis: prospects for xylene isomer separation. J Membr Sci 2009; 337: 106-112. http://dx.doi.org/10.1016/j.memsci.2009.03.028

[9] Li Y, Pera-Titus M, Xiong G, Yang W, Landrivon E, Miachon S, Dalmon J-A. Nanocomposite MFI zeolite - alumina membranes via pore-plugging synthesis: genesis of the material. J Membr Sci 2008; 325: 973-981. http://dx.doi.org/10.1016/j.memsci.2008.09.030

[10] Miachon S, Dalmon J-A. Catalysis in membrane reactors: what about the catalyst? Topics in Catalysis 2004; 29: 59-65. http://dx.doi.org/10.1023/B:TOCA.0000024928.63811.8b

[11] Miachon S, Landrivon E, Aouine M, Sun Y, Kumakiri I, Li Y, Pachtova Prokopova O, Guilhaume N, Giroir-Fendler A, Mozzanega H, Dalmon J-A. Nanocomposite MFI-alumina membranes via pore-plugging synthesis: preparation and morphological characterization. J Membr Sci 2006; 281: 228-

238.

http://dx.doi.org/10.1016/i.memsci.2006.03.036

[12] Daramola MO, Burger AJ, Pera-Titus M, Giroir-Fendler A, Lorenzen L, Dalmon J-A. Xylene vapor mixture separation in nanocomposite MFI-alumina tubular membranes: Influence of operating variables. Sep Sci Technol 2010; 45(1): 21-27. http://dx.doi.org/10.1080/01496390903402141

[13] Alshebani A, Pera-Titus M, Landrivon E, Schiestel $T$, Miachon S, Dalmon J-A. Nanocomposite MFI-ceramic hollow fibres: Prospects for $\mathrm{CO}_{2}$ separation. Micropor Mesopor Mater 2008; 115: 197-205. http://dx.doi.org/10.1016/j.micromeso.2007.11.050

[14] Bayati B, Babaluo AA, Karimi R. Hydrothermal synthesis of nanostructure NaAzeolite: The effect of synthesis parameters on zeolite seed size and crystallinity. J Eur Ceram Soc 2008; 28: 2653-2657. http://dx.doi.org/10.1016/j.jeurceramsoc.2008.03.033

[15] Naskar MK, Kundu D, Chatterjee M. Effect of process parameters on surfactant-based synthesis of hydroxy sodalite particles. Mater Lett 2011; 65: 436-438. http://dx.doi.org/10.1016/j.matlet.2010.11.008

[16] Kundu D, Dey B, Naskar MK, Chatterjee M. Emulsion-derived urchin-shaped hydroxy sodalite particles. Mater Lett 2010 64: 1630-1633. http://dx.doi.org/10.1016/j.matlet.2010.04.015

[17] Chen X, Qiao M, Xie S, Fan K, Zhou WZ, He H. Selfconstruction of core-shell and hollow zeolite analcime icositetrahedra: a reversed crystal growth process via recrystallization from surface to core. J Am Chem Soc 2007; 129: $13305-13312$ http://dx.doi.org/10.1021/ja074834u

[18] Buhl J-C, Schuster K, Robben L. Nanocrystalline sodalite grown from superalkaline $\mathrm{NaCl}$ bearing gels at low temperature $(333 \mathrm{~K})$ and the influence of TEA on crystallization process. Micropor Mesopor Mater 2011; 142: 666-671.

http://dx.doi.org/10.1016/j.micromeso.2011.01.020

[19] Thompson RW. In: Verified synthesis of zeolitic Materials, $\mathrm{H}$ Robson (Eds.), Elsevier Science 2001; pp. 21-23. http://dx.doi.org/10.1016/B978-044450703-7/50100-9 